\title{
Forces and Trauma Associated with Minimally Invasive Image-Guided Cochlear Implantation
}

Otolaryngology-

Head and Neck Surgery

20I4, Vol. I50(4) 638-645

(C) American Academy of

Otolaryngology-Head and Neck

Surgery Foundation 2014

Reprints and permission:

sagepub.com/journalsPermissions.nav DOI: I0.1 I77/01945998।3519747 http://otojournal.org

@SAGE

\author{
Pooyan Rohani, ME', Jason Pile, $\mathbf{M S}^{2}$, Lueder A. Kahrs, PhD $^{3}$, \\ Ramya Balachandran, $\mathrm{PhD}^{4}$, Grégoire S. Blachon ${ }^{4}$, \\ Nabil Simaan, PhD $^{2}$, and Robert F. Labadie, MD, PhD $^{4}$
}

Sponsorships or competing interests that may be relevant to content are disclosed at the end of this article.
Received October 23, 20I3; revised December 10, 2013; accepted December 18, 2013.

\begin{abstract}
Objective. Minimally invasive image-guided cochlear implantation $(\mathrm{Cl})$ utilizes a patient-customized microstereotactic frame to access the cochlea via a single drill-pass. We investigate the average force and trauma associated with the insertion of lateral wall $\mathrm{Cl}$ electrodes using this technique.
\end{abstract}

Study Design. Assessment using cadaveric temporal bones.

Setting. Laboratory setup.

Subjects and Methods. Microstereotactic frames for 6 fresh cadaveric temporal bones were built using CT scans to determine an optimal drill path following which drilling was performed. $\mathrm{Cl}$ electrodes were inserted using surgical forceps to manually advance the $\mathrm{Cl}$ electrode array, via the drilled tunnel, into the cochlea. Forces were recorded using a 6-axis load sensor placed under the temporal bone during the insertion of lateral wall electrode arrays (2 each of Nucleus C1422, MED-EL standard, and modified MED-EL electrodes with stiffeners). Tissue histology was performed by microdissection of the otic capsule and apical photo documentation of electrode position and intracochlear tissue.

Results. After drilling, CT scanning demonstrated successful access to cochlea in all 6 bones. Average insertion forces ranged from 0.009 to $0.078 \mathrm{~N}$. Peak forces were in the range of 0.056 to $0.469 \mathrm{~N}$. Tissue histology showed complete scala tympani insertion in 5 specimens and scala vestibuli insertion in the remaining specimen with depth of insertion ranging from $360^{\circ}$ to $600^{\circ}$. No intracochlear trauma was identified.

Conclusion. The use of lateral wall electrodes with the minimally invasive image-guided $\mathrm{Cl}$ approach was associated with insertion forces comparable to traditional $\mathrm{Cl}$ surgery. Deep insertions were obtained without identifiable trauma.

\section{Keywords}

cochlear implantation, cochlear histology, cochlear electrode, insertion forces, minimally invasive surgery

\section{Introduction}

Cochlear implants (CI) are the standard of care for treatment of severe to profound bilateral sensorineural hearing loss. The traditional surgical approach, first described by CI pioneer William House, involves mastoidectomy followed by posterior tympanotomy to provide access to the basal turn of the cochlea. This technique is well known to surgeons comfortable with facial recess anatomy. For surgeons more comfortable with endaural approaches to middle ear pathology, the suprameatal approach was developed. ${ }^{1,2}$ This approach uses anatomical measurements from the surface of the temporal bone to estimate and drill a tunnel to access the attic. Following this drilling, the middle ear is addressed via a tympanomeatal flap, an entry into the cochlea is made via either the round window (RW) or a separate cochleostomy, and the CI electrode passed from the tunnel to the attic and then threaded into the cochlea. This approach is limited to highly flexible, lateral wall electrodes and is not suitable for styleted, perimodiolar electrodes. Over 500 suprameatal approaches have been reported in the literature.

Perhaps the biggest downside to the suprameatal approach is the suboptimal insertion vector as the electrode must be redirected as it courses from the attic to the

\footnotetext{
'Vanderbilt University School of Medicine, Nashville, Tennessee, USA

${ }^{2}$ Department of Mechanical Engineering, Vanderbilt University, Nashville, Tennessee, USA

${ }^{3}$ Institute of Mechatronic Systems, Leibniz University Hannover, Hannover, Germany

${ }^{4}$ Department of Otolaryngology-Head and Neck Surgery, Vanderbilt University Medical Center, Nashville, Tennessee, USA
}

This article was presented at the 2013 AAO-HNSF Annual Meeting \& OTO EXPO; September 29-October 3, 2013; Vancouver, British Columbia, Canada.

\section{Corresponding Author:}

Robert F. Labadie, MD, PhD, Vanderbilt University Medical Center, 7209

Medical Center East, South Tower, Nashville, TN 37232-8606, USA.

Email: robert.labadie@vanderbilt.edu 
mesotympanum and ultimately into the cochlea. Capitalizing on image-guidance technology to provide a tunnel through the facial recess and preserve an optimal insertion vector, our group has developed and reported on a minimally invasive imageguided implantation technique previously called Percutaneous Cochlear Implantation (PCI). Validated in vitro ${ }^{3-5}$ and in vivo, ${ }^{6,7}$ this approach utilizes CT imaging to specify a trajectory that allows access to the cochlea via a single drill-pass from the lateral skull through the facial recess to an optimal insertion point in the scala tympani (ST) subcomponent of the cochlea. The specified trajectory is accurately drilled on patients through the use of a patient-customized, microstereotactic frame, which is affixed to the skull of the patient using bone-implanted markers that serve both as fiducial markers to register the patient to their CT scan and as mounting points for the microstereotactic frame. While accuracy for this technique has been determined to be in the submillimetric range (target registration error of the system is $0.37 \pm 0.18 \mathrm{~mm}^{5}$ ), force associated with insertion of electrode arrays with this technique has yet to be determined in comparison to traditional surgical approaches. Thus, the overall purpose of this study was to assess the forces and potential intracochlear trauma associated with the insertion of electrodes via the minimally invasive image-guided $\mathrm{CI}$ approach. More specifically, this study aims to assess the insertion impact of 3 different electrode types by analysis of intraoperative force data, postoperative electrode placement status, and intracochlear trauma outcomes via the minimally invasive image-guided CI technique.

\section{Methods}

Six fresh cadaveric temporal bones were harvested for use in this study. No Institutional Review Board approval was required, as the research involved postmortem specimens. As histological processing of the samples was an important objective of the study, fresh specimens were obtained within 120 hours of death, placed in buffered formalin, and transported. Tympanomeatal flaps were raised and the stapes removed to allow formalin to enter the inner ear to preserve structures. Bones were then refrigerated for the short interval - several days - between arrival of specimens and the experimentation described in the following.

The minimally invasive image-guided approach to access the cochlea was completed by performing the following steps on individual specimens:

Step 1: Three fiducial markers were bone-implanted in each sample at the mastoid tip, supra-helical region, and posterior auricular area (Figure I).

Step 2: CT image of the sample covering the entire specimen with the fiducial markers was acquired using an xCAT ENT mobile CT scanner (Xoran Technologies Inc, Ann Arbor, Michigan).

Step 3: Automatic segmentation of critical structures in the CT scan, including the facial nerve, the chorda typmani, and the cochlea, was performed. ${ }^{8,9}$ A safe linear drill trajectory was then automatically planned from the lateral skull to the cochlea

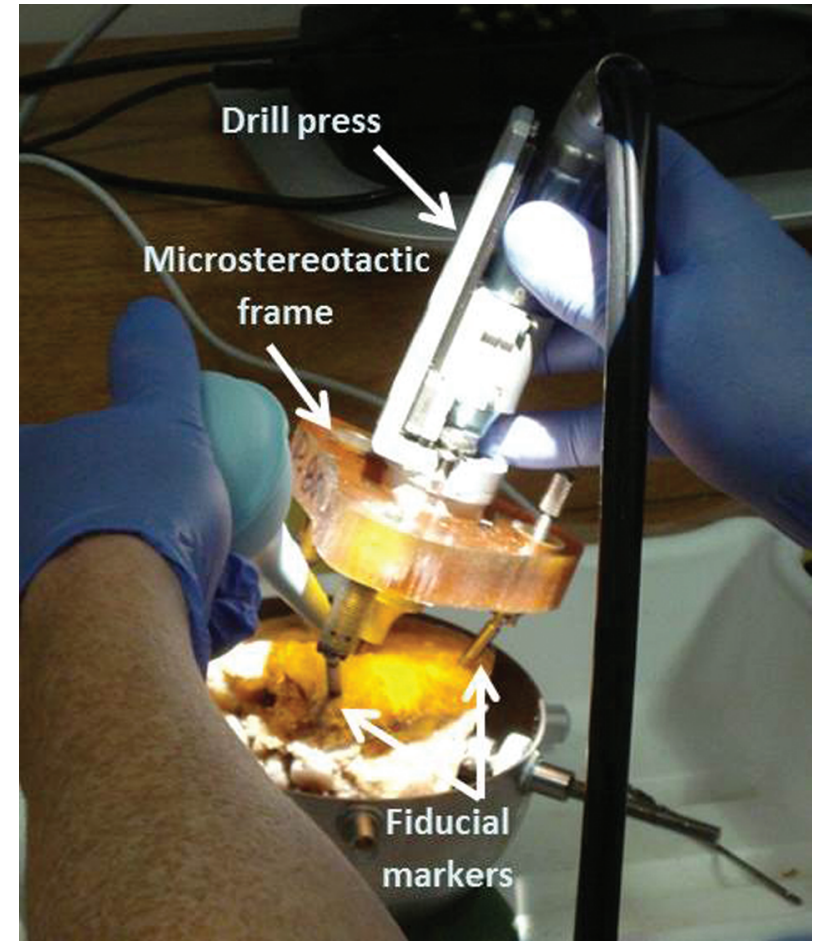

Figure I. Drilling using custom microstereotactic frame on a temporal bone specimen. Bone-implanted fiducial markers allow rigid attachment of the frame to the specimen.

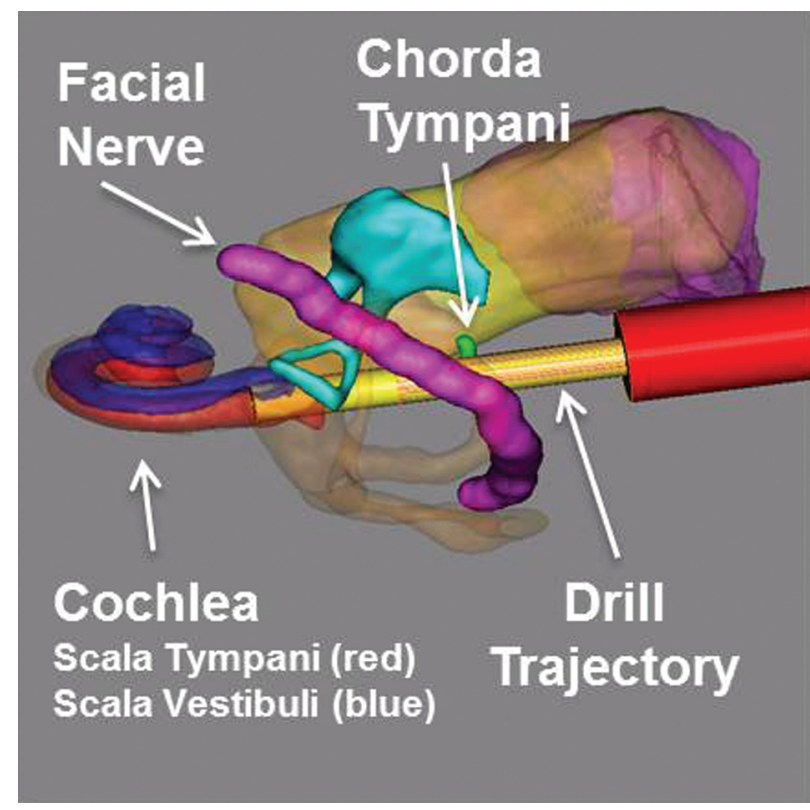

Figure 2. Three-dimensional rendering of the segmented structures and the drill path. The red cylinder along the drill path represents the determined depth for stopping the lateral drilling.

through the facial recess. ${ }^{10}$ This trajectory was independently confirmed by a study investigator for each bone specimen, and drilling depths along the path for the different diameter drill bits mentioned in step 6 were determined (Figure 2). 


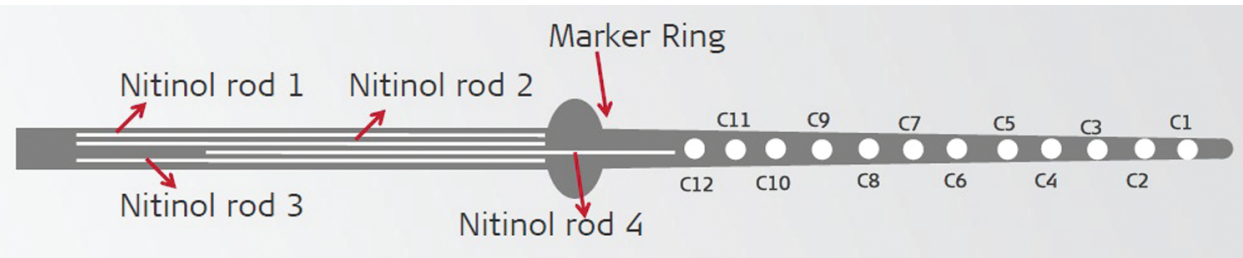

Figure 3. The design of the MED-EL custom electrode. Four nitinol stiffening rods were added in the silicone surrounding the extracochlear transmitting wire from the internal receiver to the beginning of the electrode array.

Step 4: A custom microstereotactic frame was designed and constructed for each specimen using a standard computer numeric control (CNC) milling machine. The frame was fabricated in approximately 5 minutes using data created by custom software written in Matlab (Mathworks Inc., Natick, Massachusetts) that specifies the exact dimensions necessary in order to mount the frame on the fiducial markers and drill the desired trajectory.

Step 5: To provide direct visualization to the middle ear, a tympanomeatal flap was raised and the RW overhang was drilled away to allow complete visualization of the RW membrane.

Step 6: The microstereotactic frame was installed onto the fiducial markers and was used to guide a custom manual drill press. The desired trajectory was drilled using a $3.8 \mathrm{~mm}$ twist drill bit laterally and a $1.6 \mathrm{~mm}$ twist drill bit medially. ${ }^{3}$ The wider lateral hole allows irrigation during deeper drilling and placement of a bushing for the thinner drill bit closer to the facial recess and cochlea to perform accurate drilling that will be safe to the structures. The drill press was set to limit the depth of drilling based on the measurements in the CT scan to prevent any damage to critical structures. In general, the lateral drilling was set to stop with about $5 \mathrm{~mm}$ safety distance from the facial recess and the medial drilling was set to stop in the middle ear region. A cochleostomy was then performed by replacing the twist drill bit with a $1 \mathrm{~mm}$ spherical diamond drill bit to minimize damage to the cochlea. ${ }^{11}$ Opening of the cochlea was confirmed microscopically prior to moving forward.

To ensure consistency among the force and trauma data obtained for each electrode type, the same surgeon performed all the insertions. Two of each Nucleus CI422 electrodes (Cochlear Ltd, Sydney, Australia), MED-EL standard electrodes (MED-EL GmbH, Innsbruck, Austria), and MED-EL custom electrodes with stiffeners were used in this experiment. The MED-EL custom electrodes were developed in cooperation with the manufacturer by adding 4 nitinol stiffening rods in the silicone surrounding the extracochlear transmitting wire from the internal receiver to the beginning of the electrode array (Figure 3). Prior experience had shown the standard MED-EL electrode type to be quite flexible, ${ }^{12}$ and the stiffening rods were added to assess

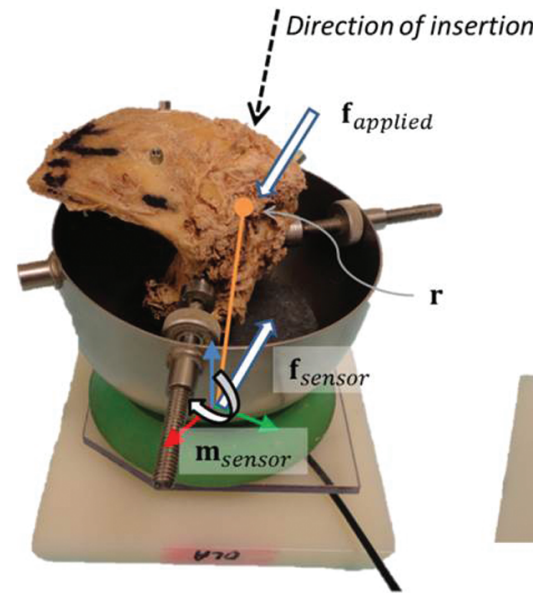

ATI Nano 43 with coordinate frame

Figure 4. Experimental setup. The temporal bone (left image) was placed on the Nano43 force sensor (right image). The sensor coordinate frame and representative illustration of the insertion direction, point of contact, forces, and moment are included.

any change in the depth of insertion by using a more rigid prototype.

Each specimen was placed on the load sensor to collect forces (Figure 4). The sensor used was a Nano43 6-axis force and moment transducer with a resolution of 0.004 Newtons (N) and sampling rate of $2 \mathrm{kHz}$ (ATI Industrial Automation Inc, Apex, North Carolina). After a specimen was placed on the surface of the sensor, the output force was tarred to zero. Any additional force onto the sensor was assumed to be applied by contact with the implant or the surgeon. Force and moment data were recorded and analyzed using custom-written MATLAB software (MathWorks).

Each insertion was monitored via 2 methods. The surgeon used a microscope placed directly above the drill hole to guide advancement of the electrode. Additionally, another view was available via an endoscope placed in the external auditory meatus and secured with an endoscope holder such that it did not contact the specimen (ie, did not contribute force to the load sensor). The endaural approach was not used at the time of the insertion other than to provide visualization that the electrode was entering the cochlea. Electrodes were inserted until the surgeon felt additional force would cause damage. At this point, a small amount of cyanoacrylate glue was applied to secure the electrode to the cochleostomy in order to limit electrode movement during the next steps. 

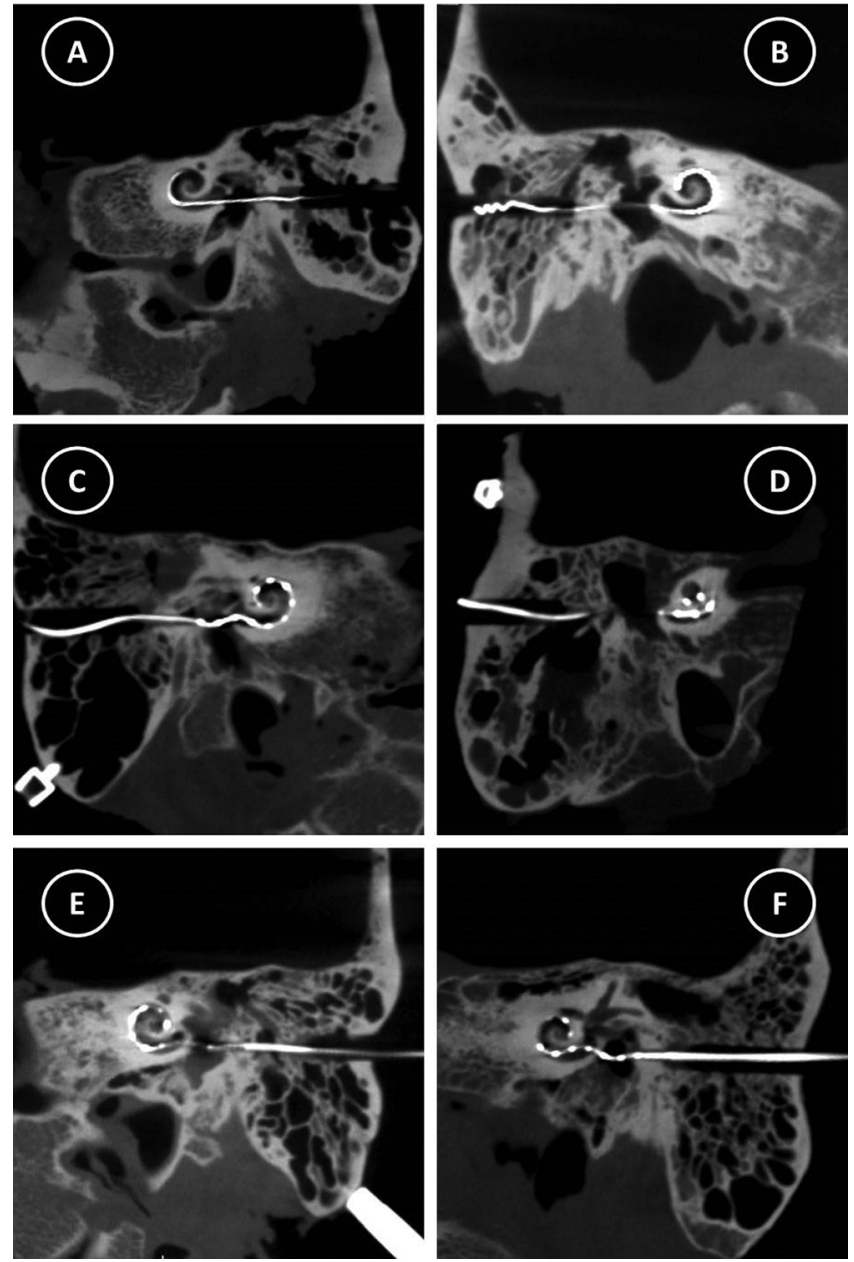

Figure 5. Postoperative CT images demonstrating electrode array insertion. (A) Specimen I, Nucleus Cl422, (B) Specimen 2, Nucleus Cl422, (C) Specimen 3, MED-EL, Standard, (D) Specimen 4, MEDEL, Standard, (E) Specimen 5, MED-EL, Custom, (F) Specimen 6, MED-EL, Custom.

Immediate post-insertion $\mathrm{CT}$ imaging was performed to assess electrode array placement. Following this step, cochleae were harvested from the temporal bones using a sagittal oscillating saw (Stryker Corporation, Kalamazoo, Michigan). The samples then underwent microdissection and staining following a procedure that has been previously detailed elsewhere. ${ }^{13}$ Briefly, the membranous labyrinth was stained with osmium tetroxide to allow for visualization of nerve fibers. The bone overlying the ST was decalcified and carefully removed followed by removal of Reissner's membrane. These steps allow a clear view of the basilar membrane and the underlying ST. The structures within the inner ear were carefully examined and photographed to assess for any structural trauma.

\section{Results}

\section{Location and Histopathology Results}

Post-insertion CT imaging revealed all 6 electrodes coursed through the cochlea (Figure 5). The facial nerve was intact
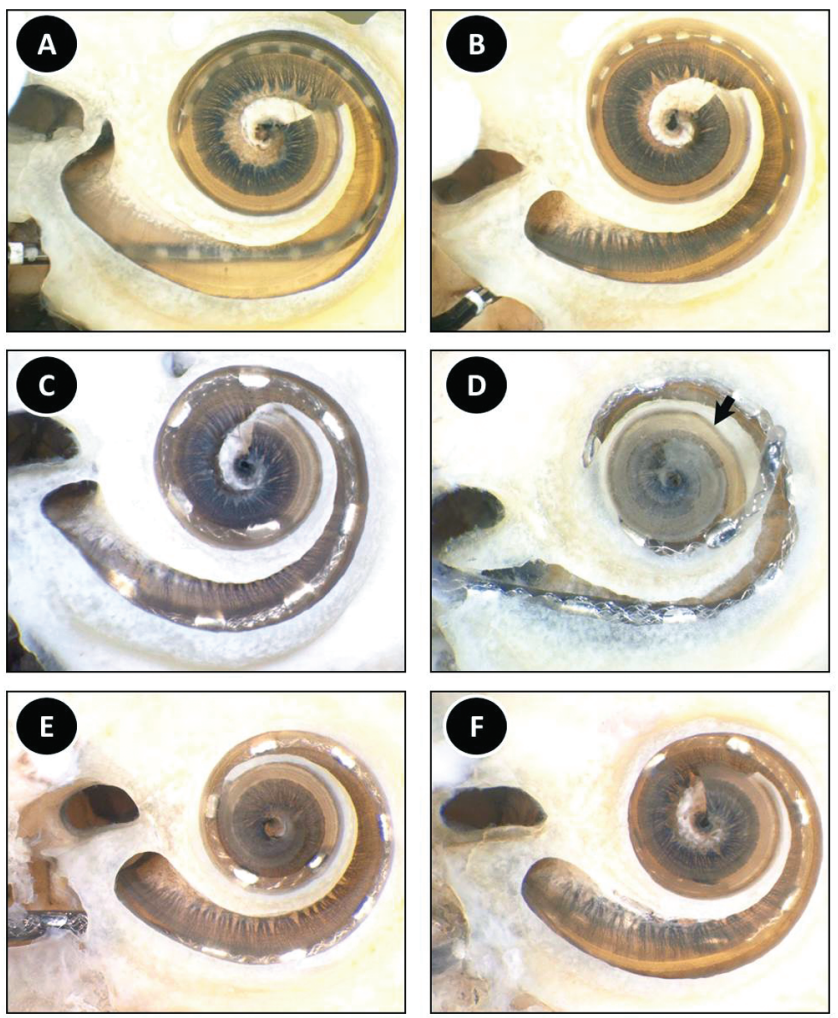

Figure 6. Histologic analysis of the post-insertion specimens. An apical view of each specimen is provided in which the overlying otic capsule is removed and decalcified osseous spiral lamina and basilar membrane allow visualization of the underlying scala tympani. The arrow in panel $D$ points to the apical-most location of the electrode prior to upward movement as a result of microdissection. (A) Specimen I, Nucleus Cl422, (B) Specimen 2, Nucleus Cl422, (C) Specimen 3, MED-EL, Standard, (D) Specimen 4, MEDEL, Standard, (E) Specimen 5, MED-EL, Custom, (F) Specimen 6, MED-EL, Custom.

after drilling in all specimens. The chorda tympani was sacrificed in 2 specimens as planned prior to drilling due to small facial recesses in those bones. It remained intact in 4 specimens. Figure $\mathbf{6}$ shows tissue histology results. An apical view of each specimen is provided in which the overlying otic capsule is removed and decalcified osseous spiral lamina and basilar membrane allow visualization of the underlying ST. Both right and left ear specimens were used in the study. However, to facilitate comparison, those of the left ears have been flipped horizontally in the histology images.

Both insertions of the Nucleus CI422 electrodes resulted in ST placement of the entire electrode array. The osseous spiral lamina and the basilar membrane were intact in both cases. Specimen 1 showed a $360^{\circ}$ insertion at the apicalmost portion (Figure 6A). The electrode was placed laterally for most of its length with the exception of the base, where it rested more medially near the modiolus. A microdefect on the lower side of the osseous spiral lamina was noted; however, this defect did not traverse through the thickness of the spiral lamina and therefore this structure 


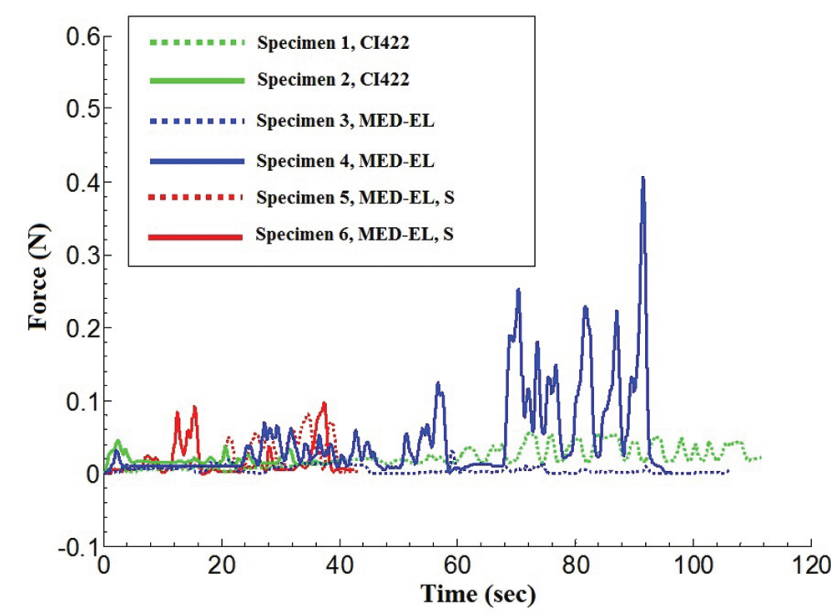

Figure 7. Insertion forces corresponding to the three electrode types.

was in place. Specimen 2 showed no evidence of trauma (Figure 6B). Similar to the previous specimen, the electrode was inserted approximately $360^{\circ}$. The electrode was placed in close proximity to the lateral wall throughout its course.

MED-EL standard electrode insertions resulted in 1 ST (10 of 12 electrodes intracochlear) and 1 scala vestibuli (SV) (11 of 12 electrodes intracochlear) placement. Specimen 3 showed trauma-free ST insertion along the lateral wall at a depth of $450^{\circ}$ (Figure 6C). In Specimen 4 the electrode was placed in the SV along the entirety of its course (Figure 6D). During the microdissection process, removal of the otic capsule resulted in upward spring-like motion of the electrode. However, the apical-most area of the electrode was placed where indicated by the black arrow. There was no visible trauma to any of the inner ear structures and the depth of insertion was approximately $600^{\circ}$.

Both MED-EL custom electrodes with stiffeners were successfully placed in the ST with both having 10 of 12 electrodes placed intracochlear. Specimen 5 showed an insertion depth of $540^{\circ}$ along the lateral wall with no visible trauma (Figure 6E). Specimen 6 showed an insertion depth of $450^{\circ}$ with no trauma (Figure $6 \mathbf{F}$ ). The electrode did appear somewhat curved at the basal turn, with deviation toward the modiolus. However, the remainder of the electrode was located laterally.

\section{Force Data}

Raw force results corresponding to each insertion are shown in Figure 7. The insertion times varied between 38 and 113 seconds. Average insertion forces were 9 to 78 milliN. Peak forces ranged from 56 to 469 milliN. The highest force and variation coincided with the insertion into the SV. This insertion also had peak forces greater than 4 times the second highest instance. It was the only insertion that showed a significant variation in the location of contact. Location of contact, expected moment, and a weighted average were calculated, described in the following, to account for any forces not applied by the implant onto the cochlea.

The experimental setup records all forces acting on the temporal bone, which may include forces applied by the insertion tool or the surgeon. The measured force data must be checked for consistency so that reported forces reflect only those applied by the implant onto the cochlea. The location of the cochlea with respect to the sensor was not known a priori. However, the location of force applied to the cochlea by the electrode was restricted to a relatively small volume, and this information was used to detect outliers - forces not applied by the electrode. Assuming that the insertion of the electrode, with a highly flexible structure, does not impart significant moments about the $\mathrm{x}, \mathrm{y}$, or $\mathrm{z}$ axes, the sensed force and moment data can be used to determine the average location of contact in the force sensor frame. Expected moment can then be calculated. Any significant difference between the expected and measured moment, referred to by residual moment, $m_{\text {residual }}$, indicates periods of time when other forces may be acting on the bone. For example, significant contact between the surgical forceps and the specimen can be identified by this method. The moment sensor has a resolution of $0.1 \mathrm{~N}-\mathrm{m}\left(m_{\text {error }}\right)$. If $m_{\text {residual }}$ was larger than $m_{\text {error }}$, a correction factor, or weight, ranging between 0 and 1 was applied to the corresponding force measurement. The weight $(w)$ is defined as:

$$
w=e^{-\alpha\left(\frac{m_{\text {residual }}-m_{\text {error }}}{m_{\text {error }}}\right)} \text { for } m_{\text {residual }}>m_{\text {error }} .
$$

In this equation, when $\alpha$ is set to 0.35 , the assigned weight to a particular force would be half the original value since the residual moment outcome is twice the magnitude of the expected error. In effect, value of $\alpha$ determines how quickly confidence is lost in that a particular measured force corresponded purely to electrode insertion.

Average, weighted average, and peak forces corresponding to histological outcomes noted in each specimen are presented in Table I. While unweighted averages for each insertion include all force data points, weighted averages correct for outlier data as described previously. Of note, reported peak forces do not include data points corrected by the aforementioned weighting formula.

\section{Discussion}

Using the minimally invasive image-guided surgical approach, we successfully gained access to the cochlea and inserted electrodes in 6 out of 6 specimens with minimal trauma. Five of the 6 specimens were inserted fully in ST and 1 of the 6 inserted fully in SV. The only visible trauma was a micro-defect on the lower side of the osseous spiral lamina, which did not traverse the thickness of the spiral lamina in the first Nucleus CI422 insertion. Regarding the insertion in SV, comparison of the planned path with the actual drilled path as identified on the post-insertion CT showed a minor deviation of the drilled path from the planned trajectory during the drilling process, resulting in 
Table I. Force and histology outcomes summary.

\begin{tabular}{llccccc}
\hline Specimen & Electrode & Average force $(\mathrm{N})$ & Weighted average force (N) & Peak force (N) & Insertion angle & Location \\
\hline I & Cochlear 422 & 0.023 & 0.022 & 0.056 & $360^{\circ}$ & ST \\
2 & Cochlear 422 & 0.043 & 0.043 & 0.067 & $360^{\circ}$ & ST \\
3 & MED-EL & 0.009 & 0.008 & 0.061 & $450^{\circ}$ & ST \\
4 & MED-EL & 0.078 & 0.053 & 0.469 & $600^{\circ}$ & SV \\
5 & MED-EL, S & 0.023 & 0.023 & 0.084 & $540^{\circ}$ & ST \\
6 & MED-EL, S & 0.020 & 0.020 & 0.105 & $450^{\circ}$ & ST \\
\hline
\end{tabular}

Abbreviations: ST, scala tympani; SV, scala vestibuli.

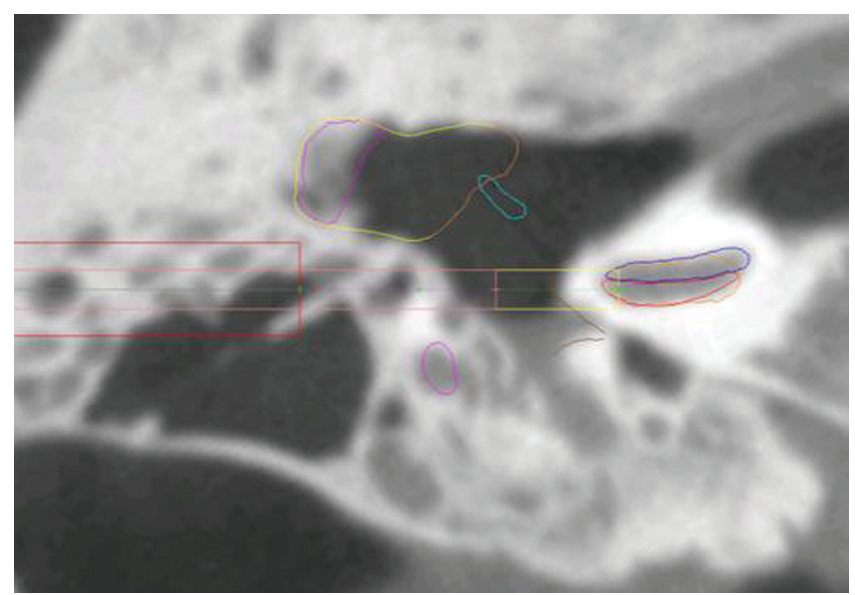

Figure 8. CT scan of specimen 4. The large air cell present during the transition from the wide to thin drill bit may have caused the thin drill bit to deviate on hitting the hard bone surface.

the targeting of the SV. Possible explanations for this targeting error include: (1) error in localizing the location of the fiducial markers due to poor image quality of the flat panel cone beam CT scanner used for this study, ${ }^{14}$ (2) error in assembling the microstereotactic frame, and/or (3) deviation by the drill bit on contact with the bone. Based on our prior studies, ${ }^{3-7}$ we believe poor image quality or unstable microstereotactic frame assembly were unlikely causes of deviation. A closer look at the CT scan of the specimen reveals the presence of a large air cell measuring approximately 6 $\times 10 \times 13 \mathrm{~mm}^{3}$ within which the $3.8 \mathrm{~mm}$ drill was set to stop (Figure 8). Following the switch to the $1.6 \mathrm{~mm}$ drill bit, advancement and eventual contact with the bone could have potentially caused the bit to deviate and follow a slightly different path, even despite the use of a bushing to constrain movement. The ultimate drilled path was away from the facial nerve and did not cause damage to it.

In both temporal bone models and clinical reports, intracochlear trauma secondary to $\mathrm{CI}$ is relatively common occurring clinically in as many as one-third of CI insertions. ${ }^{15}$ The minimally invasive image-guided approach does provide an optimal insertion angle specified as that angle which allows a $10 \mathrm{~mm}$ long cylinder to be placed into the basal turn of the ST. The drilled tunnel directs insertion forces coaxial with this insertion angle and, theoretically, results in less intracochlear trauma. While this less trauma effect was noted in the current study, we also note that for those cases in which resistance was met prior to complete insertion, buckling of the electrode array in the basal turn was noted and can be seen in the post-insertion CT scans of specimens 3 and 6 .

The electrode array with the smallest diameter, the Nucleus CI422, was completely inserted, namely, all electrodes intracochlear, in both cases to a depth of $360^{\circ}$. While the standard MED-EL arrays had deeper insertions ranging from $450^{\circ}$ to $600^{\circ}$, because of their geometry, incomplete insertion was achieved with only 10 to 11 of the 12 electrodes intracochlear. The MED-EL custom electrodes with stiffeners were developed specifically for this surgical approach to allow use of the stiffened transmitting wire (from the internal receiver to the electrode array) as an insertion tool that the surgeon could grasp and insert without the use of additional instruments. While this modified electrode worked as designed, the depth of insertion was not dramatically different than that of the standard MED-EL electrodes without the stiffeners.

Peak insertion forces, with the exception of the SV placement in specimen 4, were at or below 105 milliN with an average force of approximately $30 \mathrm{milliN}$ across the studied electrodes. Previous studies of forces associated with electrode insertions in the setting of traditional CI have varied greatly from less than 5 milliN to 200 milliN using both lateral wall and perimodiolar electrodes. Insertions were performed in either bone samples or cochlear models. A summary of the most relevant studies is included as Table 2. ${ }^{16-20}$ The average forces obtained in the current study are comparable with the range of those previously reported.

\section{Conclusion}

We presented the force and trauma associated with the insertion of electrode arrays via the minimally invasive image-guided CI approach, which involves inserting an electrode array via a narrow linear tunnel drilled from the lateral skull to the cochlea. We have shown on 6 cadaveric temporal bone specimens that deep insertion was possible without crossover and without identifiable trauma using 3 different lateral wall electrodes. The average insertion forces measured in our study were comparable to the reported average insertion forces for traditional CI approach. 
Table 2. Previously published average forces of insertion in traditional cochlear implantation.

\begin{tabular}{llll}
\hline Lead author & Average force $(\mathrm{N})$ & \multicolumn{1}{c}{ Electrode } & Target \\
\hline Roland $^{16}$ & $0.15-0.2(\mathrm{SIT})$ & Cochlear, NCA (PM) & Temporal bone \\
& $<0.05(\mathrm{AOS})$ & & \\
Schurzig $^{17}$ & $0.046 \pm 0.027$ & Cochlear, Freedom (PM) & Cochlear model \\
Majdani $^{18}$ & $0.004 \pm 0.001$ & Cochlear, NCA (PM) & Cochlear model \\
Miroir $^{19}$ & $<0.20$ & Advanced Bionics, IJ (LW) & Temporal bone \\
Pile $^{20}$ & $<0.14$ & Cochlear, NCA (PM) & Temporal bone \\
\hline
\end{tabular}

Abbreviations: SIT, Standard Insertion Technique; AOS, Advance Off Stylet; NCA, Nucleus Contour Advance; PM, perimodiolar electrode; LW, Lateral Wall electrode.

\section{Acknowledgments}

The authors would like to sincerely thank C. Gary Wright, $\mathrm{PhD}$, and Louis Kratchman, for their contributions to and support for this work.

\section{Author Contributions}

Pooyan Rohani, drafting of manuscript, experimental design, conduct of experiment, data analysis, critical revision; Jason Pile, drafting of manuscript, experimental design, conduct of experiment, data analysis, critical revision; Lueder A. Kahrs, conduct of experiment; Ramya Balachandran, experimental design, conduct of experiment, data analysis, critical revision; Grégoire S. Blachon, conduct of experiment; Nabil Simaan, experimental design, critical revision; Robert F. Labadie, experimental design, conduct of experiment, critical revision.

\section{Disclosures}

Competing interests: Jason Pile, research in the area of robotics for cochlear implantation is funded by Cochlear Ltd; Nabil Simaan, patents licensed to Auris Surgical Robotics Inc in the area of robotics cochlear implantation, research in the area of robotics for cochlear implantation is funded by Cochlear Ltd; Robert F. Labadie, consultant, Cochlear, Corp, consultant, Medel, Corp, consultant, Medtonic, Corp, consultant, Otoronix, LLC (Note: No relationships relevant to current study), patent applications on various components of the technology pending.

Sponsorships: None.

Funding source: Research reported in this project was supported by NIH/NIDCD awards R01DC008408 and R01DC10184 and NIH/NCATS award UL1 TR000445. The content is solely the responsibility of the authors and does not necessarily represent the official views of the NIDCD, the NCATS, or the NIH.

\section{References}

1. Kronenberg J, Migirov L, Dagan T. Suprameatal approach: new surgical approach for cochlear implantation. J Laryngol Otol. 2001;115:283-285.

2. Kronenberg J, Baumgartner W, Migirov L, Dagan T, Hildesheimer M. The suprameatal approach: an alternative surgical approach to cochlear implantation. Otol Neurotol. 2004; 25:41-44.

3. Balachandran R, Mitchell JE, Blachon G, et al. Percutaneous cochlear implant drilling via customized frames: an in vitro study. Otolaryngol Head Neck Surg. 2010;142:421-426.
4. Warren FM, Balachandran R, Fitzpatrick JM, Labadie RF. Percutaneous cochlear access using bone-mounted, customized drill guides: demonstration of concept in vitro. Otol Neurotol. 2007;28:325-329.

5. Labadie RF, Mitchell J, Balachandran R, Fitzpatrick JM. Customized, rapid-production microstereotactic table for surgical targeting: description of concept and in vitro validation. Int J Comput Assist Radiol Surg. 2009;4:273-280.

6. Labadie RF, Noble JH, Dawant BM, Balachandran R, Majdani O, Fitzpatrick JM. Clinical validation of percutaneous cochlear implant surgery: initial report. Laryngoscope. 2008;118:10311039.

7. Labadie RF, Balachandran R, Mitchell JE, et al. Clinical validation study of percutaneous cochlear access using patientcustomized microstereotactic frames. Otol Neurotol. 2010;31: 94-99.

8. Noble JH, Warren FM, Labadie RF, Dawant BM. Automatic segmentation of the facial nerve and chorda tympani in CT images using spatially dependent feature values. Med Phys. 2008;35:5375-5384.

9. Noble JH, Dawant BM, Warren FM, Labadie RF. Automatic identification and 3D rendering of temporal bone anatomy. Otol Neurotol. 2009;30:436-442.

10. Noble JH, Majdani O, Labadie RF, Dawant B, Fitzpatrick JM. Automatic determination of optimal linear drilling trajectories for cochlear access accounting for drill-positioning error. Int $J$ Med Robot. 2010;6:281-290.

11. Kratchman LB, Schurzig D, McRackan TR, et al. A manually operated, advance off-stylet insertion tool for minimally invasive cochlear implantation surgery. IEEE Trans Biomed Eng. 2012;59:2792-2800.

12. McRackan TR, Balachandran R, Blachon GS, et al. Validation of minimally invasive, image-guided cochlear implantation using Advanced Bionics, Cochlear, and Medel electrodes in a cadaver model. Int J Comput Assist Radiol Surg. 2013;8:989995.

13. Wright CG, Roland PS. Temporal bone microdissection for anatomic study of cochlear implant electrodes. Cochlear Implants Int. 2005;6:159-168.

14. Balachandran R, Schurzig D, Fitzpatrick JM, Labadie RF. Evaluation of portable CT scanners for otologic image-guided surgery. Int J Comput Assist Radiol Surg. 2012;7:315-321. 
15. Wanna GB, Noble JH, McRackan TR, et al. Assessment of electrode placement and audiological outcomes in bilateral cochlear implantation. Otol Neurotol. 2011;32:428-432.

16. Roland JT, Jr. A model for cochlear implant electrode insertion and force evaluation: results with a new electrode design and insertion technique. Laryngoscope. 2005;115:1325-1339.

17. Schurzig D, Webster RJ, III, Dietrich MS, Labadie RF. Force of cochlear implant electrode insertion performed by a robotic insertion tool: comparison of traditional versus Advance OffStylet techniques. Otol Neurotol. 2010;31:1207-1210.

18. Majdani O, Schurzig D, Hussong A, et al. Force measurement of insertion of cochlear implant electrode arrays in vitro: comparison of surgeon to automated insertion tool. Acta Otolaryngol. 2010;130:31-36.

19. Miroir M, Nguyen Y, Kazmitcheff G, Ferrary E, Sterkers O, Grayeli AB. Friction force measurement during cochlear implant insertion: application to a force-controlled insertion tool design. Otol Neurotol. 2012;33:1092-1100.

20. Pile J, Simaan N. Characterization of friction and speed effects and methods for detection of cochlear implant electrode tip fold-over. In: IEEE International Conference on Robotics and Automation; 2013. 